\title{
BRAUER GROUP OF FIBRATIONS AND SYMMETRIC PRODUCTS OF CURVES
}

\author{
GEORGES ELENCWAJG
}

\begin{abstract}
Given a holomorphic fibering with fibre $\mathbf{P}_{n}$, we compare the cohomological Brauer group of the base to that of the total space. This allows us to prove that the geometric Brauer group of any symmetric product of a Riemann surface coincides with the cohomological one.
\end{abstract}

Grothendieck has introduced [GROT] the notion of a geometric Brauer group. Given a variety $X$, this group $\operatorname{Br}(X)$ classifies, roughly speaking, $\mathbf{P}_{n}$-bundles over $X$ modulo those bundles of the form $\mathbf{P}(E)$ for some vector bundle $E$. In this paper we work in the category of complex manifolds (Grothendieck's setting was, of course, scheme-theoretic). Given any manifold $Z$, we put

$$
\operatorname{Br}^{\prime}(Z):=H^{2}\left(Z, \mathcal{O}_{Z}^{*}\right)_{\text {tors }}\left(\text { torsion part of } H^{2}\left(Z, \mathcal{O}_{Z}^{*}\right)\right)
$$

The Brauer group $\operatorname{Br}(Z)$ can be identified with a subgroup of $\operatorname{Br}^{\prime}(Z)$ and a fundamental question is: does the equality $\operatorname{Br}(Z)=\operatorname{Br}^{\prime}(Z)$ hold?

The aim of this paper is to give a positive answer to this question in the following cases (Theorems 1 and 2, respectively):

(1) When $Z$ is a $\mathbf{P}_{n}$-bundle over a manifold $X$ with the property $\operatorname{Br}(X)=\operatorname{Br}^{\prime}(X)$;

(2) When $Z$ is a symmetric product $C^{(n)}$ of a compact Riemann surface.

$\S 1$ is devoted to some preliminary material. In $\S \S 2$ and 3 we prove the two theorems mentioned above.

It is my pleasant duty to thank A. Fujiki for his judicious comments and the friendly interest he took in this work.

1. We work in the category of holomorphic manifolds. A $\mathbf{P}_{r-1}$-bundle over the manifold $X$ is a holomorphic submersion $\pi: P \rightarrow X$ such that all fibres of $\pi$ are isomorphic to $\mathbf{P}_{r-1}$. Such a map is automatically a locally trivial fibration with structure group $\operatorname{PGL}(r, \mathbf{C})$.

Hence the set of isomorphism classes of such $\mathbf{P}_{r-1}$-bundles, denoted by Project $_{r-1}(X)$, is in natural bijection with $H^{1}(X, \operatorname{PGL}(r, \mathcal{O}))$. We have an exact sequence of sheaves of (noncommutative) groups on $X$,

$$
1 \rightarrow \mathcal{O}_{X}^{*} \rightarrow \operatorname{GL}\left(r, \mathcal{O}_{X}\right) \rightarrow \operatorname{PGL}\left(r, \mathcal{O}_{X}\right) \rightarrow 1,
$$

Received by the editors June 11, 1984 and, in revised form, September 26, 1984.

1980 Mathematics Subject Classification. Primary 14C30; Secondary 32L05, 14F25, 14 H15. 
yielding a map

$$
H^{1}\left(X, \operatorname{PGL}\left(r, \mathcal{O}_{X}\right)\right) \stackrel{\delta_{r}}{\rightarrow} H^{2}\left(X, \mathcal{O}_{X}^{*}\right) .
$$

In analogous fashion we have an exact sequence (where $\mu_{r}$ is the constant sheaf of $r$ th roots of unity)

$$
1 \rightarrow \mu_{r} \rightarrow \operatorname{SL}\left(r, \mathcal{O}_{X}\right) \rightarrow \operatorname{PGL}\left(r, \mathcal{O}_{X}\right) \rightarrow 1
$$

yielding a map

$$
H^{1}\left(X, \operatorname{PGL}\left(r, \mathcal{O}_{X}\right)\right) \stackrel{\varepsilon_{r}}{\rightarrow} H^{2}\left(X, \mu_{r}\right) .
$$

The map $\boldsymbol{\delta}_{r}$ (resp. $\boldsymbol{\varepsilon}_{r}$ ) is to be interpreted as the obstruction for a $\mathbf{P}_{r-1}$-bundle to be of the form $\mathbf{P}(E)$ for some holomorphic vector bundle $E$ (resp. for some holomorphic vector bundle $E$ with holomorphically trivial determinant $\wedge^{r} E$ ).

These maps are related by the commutative diagram

$$
H^{1}(X, \operatorname{PGL}(r, \mathcal{O}))=\left.\operatorname{Project}_{r-1}(X) \overbrace{\delta_{r}}^{\sum_{r}^{\varepsilon_{r}} H^{2}\left(X, \mathcal{O}_{X}^{*}\right)}\right|^{H^{2}\left(j_{r}\right)} H^{2}\left(X, \mu_{r}\right)
$$

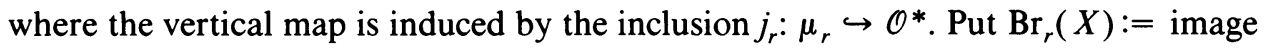
of $\delta_{r}$.

Because of the above diagram, $\mathrm{Br}_{r}(X)$ is included in the $r$-torsion part of $H^{2}\left(X, \mathcal{O}_{X}^{*}\right)$.

Define $\operatorname{Br}(X):=\bigcup_{r \in \mathrm{N}^{*}} \operatorname{Br}_{r}(X)$ and call this the Brauer group of $X$. (Motivation for this definition and further information about the results recalled above can be found in [ELEN].) The Brauer group of $X$ is included in the torsion part $\operatorname{Br}^{\prime}(X)=$ $H^{2}\left(X, \mathcal{O}_{X}^{*}\right)_{\text {tors }}$ ("cohomological Brauer group") of $H^{2}\left(X, \mathcal{O}_{X}^{*}\right)$. The principal question in this theory is whether $\operatorname{Br}(X)$ equals $\operatorname{Br}^{\prime}(X)$.

The following easy remark exhibits a situation where equality holds.

Remark 1. Let $f: Z \rightarrow X$ be a holomorphic map between manifolds. Assume the induced map $H^{2}\left(X, \mathcal{O}_{X}^{*}\right)_{\text {tors }} \rightarrow H^{2}\left(Z, \mathcal{O}_{Z}^{*}\right)_{\text {tors }}$ is surjective. Then we have the implication

$$
\operatorname{Br}(X)=\operatorname{Br}^{\prime}(X) \Rightarrow \operatorname{Br}(Z)=\operatorname{Br}^{\prime}(Z) .
$$

Proof. Suppose $\alpha \in H^{2}\left(Z, \mathcal{O}_{Z}^{*}\right)_{\text {tors }}$. The surjectivity assumption shows that $\alpha$ is the image of some $\beta \in H^{2}\left(X, \mathcal{O}_{X}^{*}\right)_{\text {tors }}$. If $\operatorname{Br}(X)=\mathrm{Br}^{\prime}(X), \beta \in \mathrm{Br}_{r}(X)$ for some $r$, i.e., $\beta=\delta_{r}(Q)$ for some $Q \in \operatorname{Project}_{r-1}(X)$. Then $\alpha=\delta_{r}\left(f^{*}(Q)\right)$ belongs to $\operatorname{Br}_{r}(Z)$. Q.E.D.

Now we give a condition under which the surjectivity assumption in Remark 1 holds.

REMARK 2. Let $f: Z \rightarrow X$ be a holomorphic map between manifolds. If, for all $r \geqslant 1$, the natural maps $H^{2}\left(X, \mu_{r}\right) \rightarrow H^{2}\left(Z, \mu_{r}\right)$ are surjective, the natural map $H^{2}\left(X, \mathcal{O}_{X}^{*}\right)_{\text {tors }} \rightarrow H^{2}\left(Z, \mathcal{O}_{X}^{*}\right)_{\text {tors }}$ is also surjective. 
Proof. Let $\operatorname{Br}_{r}^{\prime}(X)$ denote the image of the map $H^{2}\left(j_{r}\right): H^{2}\left(X, \mu_{r}\right) \rightarrow H^{2}\left(X, \mathcal{O}_{X}^{*}\right)$ induced by the inclusion $j_{r}: \mu_{r} \hookrightarrow \mathcal{O}_{X}^{*}$. The Kummer exact sequence

$$
1 \rightarrow \mu_{r} \stackrel{j_{r}}{\rightarrow} \mathcal{O}^{*} \stackrel{(.)^{r}}{\rightarrow} \mathcal{O}^{*} \rightarrow 1
$$

yields the exact sequence

$$
H^{2}\left(X, \mu_{r}\right) \stackrel{H^{2}\left(j_{r}\right)}{\rightarrow} H^{2}\left(X, \mathcal{O}_{X}^{*}\right) \stackrel{(.)^{r}}{\rightarrow} H^{2}\left(X, \mathcal{O}_{X}^{*}\right),
$$

which proves $\mathrm{Br}_{r}^{\prime}(X)=H^{2}\left(X, \mathcal{O}_{X}^{*}\right)_{r \text {-tors }}$. Consideration of the following commutative diagram then shows the result:

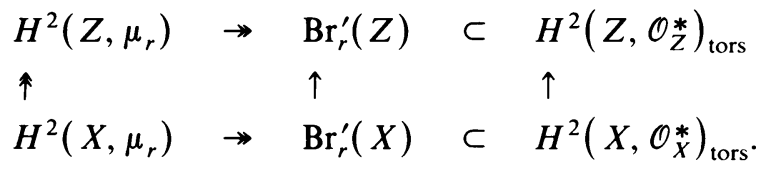

Let us end this paragraph on a note of caution.

Warning. We always have $\operatorname{Br}_{r}(X) \subset \operatorname{Br}_{r}^{\prime}(X)$. The equality $\operatorname{Br}(X)=\operatorname{Br}^{\prime}(X)$ means $\bigcup_{r} \operatorname{Br}_{r}(X)=\bigcup_{r} \operatorname{Br}_{r}^{\prime}(X)$, but does not imply the individual equalities $\operatorname{Br}_{r}(X)=$ $\operatorname{Br}_{r}^{\prime}(X)$.

2. This section is devoted to proving

Theorem 1. Let $\pi: P \rightarrow X$ be a $\mathbf{P}_{n}$-bundle defined on the complex manifold $X$. Denote by $[P]$ the obstruction class $[P]=\delta_{n+1}(P)$. Then the map $\pi$ induces, by lifting cohomology classes, an isomorphism $\mathrm{Br}^{\prime}(P) \simeq \operatorname{Br}^{\prime}(X) / Z$. $[P]$.

Proof. We are interested in $\operatorname{Br}^{\prime}(P)=H^{2}\left(P, \mathcal{O}_{P}^{*}\right)_{\text {tors }}$, so we first study the whole group $H^{2}\left(P, \mathcal{O}_{P}^{*}\right)$.

The natural tool is Leray's spectral sequence

$$
E_{2}^{p q}=H^{p}\left(X, R^{q} \pi_{*} \mathcal{O}_{P}^{*}\right) \Rightarrow H^{p+q}\left(P, \mathcal{O}_{P}^{*}\right) .
$$

Since $H^{q}\left(\mathbf{P}_{n}, \mathcal{O}\right)=0$ for $q \geqslant 1$, we deduce

$$
R^{q} \pi_{*} \mathcal{O}_{P}=0 \text { for } q \geqslant 1 .
$$

Also, $H^{q}\left(\mathbf{P}_{n}, \mathbf{Z}\right)=0$ if $q$ is odd or if $q$ is even and $q>2 n$, so

$$
R^{q} \pi_{*} \mathbf{Z}=0 \text { if } q \text { is odd or if } q \text { is even and } q>2 n \text {. }
$$

If $q$ is even and $q \leqslant 2 n$, the sheaf $R^{q} \pi_{*} \mathrm{Z}$ is a local system of coefficients with stalk $\mathbf{Z}$. The complex structure of the fibres of $\pi$ provides us with a canonical positive generator for each stalk of this local system.

Hence, $R^{q} \pi_{*} \mathbf{Z}$ is trivial: $R^{q} \pi_{*} \mathbf{Z} \simeq \mathbf{Z}$.

Summing up, we have calculated

$$
R^{q} \pi_{*} \mathbf{Z}\left\{\begin{array}{l}
=0 \quad \text { if } q \text { is odd or if } q \text { is even and } q>2 n, \\
\simeq \mathbf{Z} \text { if } q \text { is even and satisfies } 0 \leqslant q \leqslant 2 n .
\end{array}\right.
$$

The exponential sequence $0 \rightarrow \mathbf{Z} \rightarrow \mathcal{O}_{P} \rightarrow \mathcal{O}_{P}^{*} \rightarrow 1$ on $P$ leads to the long exact sequence on $X$ :

$$
\cdots \rightarrow R^{q} \pi_{*} \mathcal{O}_{p} \rightarrow R^{q} \pi_{*} \mathcal{O}_{P}^{*} \rightarrow R^{q+1} \pi_{*} \mathbf{Z} \rightarrow R^{q+1} \pi_{*} \mathcal{O}_{P} \rightarrow \cdots
$$


This shows, with the help of $(*)$, that we have isomorphisms

$$
R^{q} \pi_{*} \mathcal{O}_{P}^{*} \simeq R^{q+1} \pi_{*} Z \text { for } q \geqslant 1 .
$$

Obviously, $R^{0} \pi_{*} \mathcal{O}_{P}^{*} \simeq \mathcal{O}_{X}^{*}$, so invoking $(* *)$, we get

$$
R^{0} \pi_{*} \mathcal{O}_{P}^{*} \begin{cases}\simeq \mathcal{O}_{X}^{*}, & \\ \simeq \mathbf{Z} & \text { for } q=1,3,5, \ldots, 2 n-1, \\ =0 & \text { for } q \neq 0,1,3,5, \ldots, 2 n-1\end{cases}
$$

We can now use Leray's spectral sequence mentioned at the beginning of the proof. A moderate knowledge of this technique shows the existence of the exact sequence

$$
\begin{aligned}
1 \rightarrow H^{1}\left(X, \mathcal{O}_{X}^{*}\right) \stackrel{H^{1}(\pi)}{\rightarrow} H^{1}\left(P, \mathcal{O}_{P}^{*}\right) & \stackrel{c}{\rightarrow} H^{0}(X, \mathbf{Z}) \stackrel{d}{\rightarrow} H^{2}\left(X, \mathcal{O}_{X}^{*}\right) \\
& \stackrel{H^{2}(\pi)}{\rightarrow} H^{2}\left(P, \mathcal{O}_{P}^{*}\right) \rightarrow H^{1}(X, \mathbf{Z}) .
\end{aligned}
$$

(The sheaf $\mathbf{Z}$ that occurs in $H^{0}(X, \mathbf{Z})$ and $H^{1}(X, \mathbf{Z})$ stands for $R^{1} \pi_{*} \mathcal{O}_{P}^{*}$, in accordance with $(* * *)$.)

The maps $H^{1}(\pi)$ and $H^{2}(\pi)$ are to be interpreted as liftings of line bundles and 2-cohomology classes, respectively. The map $c$ gives the relative first Chern class of a line bundle on $P$. Since $H^{0}(X, \mathbf{Z}) \simeq \mathbf{Z}$ (we assume $X$ to be connected), the map $d$ really chooses an element in $H^{2}\left(X, \mathcal{O}_{X}^{*}\right)$ : it is the obstruction element $\delta_{n+1}(P)$ of the $\mathbf{P}_{n}$-bundle $P \rightarrow X$. We have thus shown that $H^{2}(\pi)$ induces an injective morphism $\operatorname{Br}^{\prime}(X) / \mathbf{Z} \cdot[P] \rightarrow \operatorname{Br}^{\prime}(P)$.

We can now finish proving our theorem. Consider a torsion element $\alpha \in$ $H^{2}\left(P, \mathcal{O}_{P}^{*}\right)$. Since $H^{1}(X, \mathbf{Z})$ has no torsion (this is true for any topological space), we can write $\alpha=H^{2}(\pi) \beta$ for some $\beta \in H^{2}\left(X, \mathcal{O}_{X}^{*}\right)$.

Claim. $\beta$ is a torsion element.

Indeed, the relative canonical bundle $\omega_{P / X}=\wedge^{n} \Omega_{P / X}^{1}$ corresponds to an element in $H^{1}\left(P, \mathcal{O}_{P}^{*}\right)$ satisfying $c\left(\omega_{P / X}\right)=-(n+1) \in H^{0}(X, \mathbf{Z})=\mathbf{Z}$, so the image of the map $d: H^{0}(X, \mathbf{Z}) \rightarrow H^{2}\left(X, \mathcal{O}_{X}^{*}\right)$ is of $(n+1)$-torsion. If $s$ is an integer such that $s \alpha=0$, the element $s \beta$ has zero image under $H^{2}(\pi)$, so it is in the image of $d$, and we have $(n+1) s \beta=0$. Our claim is proved.

We have thus shown that the map $H^{2}\left(X, \mathcal{O}_{X}^{*}\right)_{\text {tors }} \rightarrow H^{2}\left(P, \mathcal{O}_{P}^{*}\right)_{\text {tors }}$ is surjective, and this proves Theorem 1 .

Corollary. If $\operatorname{Br}(X)=\operatorname{Br}^{\prime}(X)$, then $\operatorname{Br}(P)=\operatorname{Br}^{\prime}(P)$.

Proof. This follows from Remark 1.

As the referee kindly pointed out to me, this result was proved (in an algebraic setup) by Gabber in his thesis [GAB].

Generalizations. Obviously the above method of proof works in much more general situations. The stated version suffices to prove Theorem 2 , so we leave it to the interested reader to formulate adequate generalizations. 
3. In this section we consider a compact Riemann surface or, equivalently, a smooth complete connected algebraic curve $C$ over the field $\mathbf{C}$. As is well known, the $n$th symmetric power $C^{(n)}$ of $C$ is a smooth algebraic manifold. We prove

THEOREM 2. For every compact Riemann surface $C$ and any $n \geqslant 1$, the equality $\operatorname{Br}\left(C^{(n)}\right)=\operatorname{Br}^{\prime}\left(C^{(n)}\right)$ holds.

Proof. Let $g$ denote the genus of $C$.

First step. We assume $n \geqslant 2 g-1$. Then there is a natural holomorphic map $\pi$ : $C^{(n)} \rightarrow \operatorname{Pic}_{n}(C)$ of $C^{(n)}$ onto the piece of the Picard variety of $C$ consisting of line bundles of degree $n$ (the fibre of $\pi$ over $L$ is $\mathbf{P}(\Gamma(C, L))$ ). This map $\pi$ exhibits $C^{(n)}$ as a $\mathbf{P}_{n-g}$-bundle over a torus. Since $\mathrm{Br}=\mathrm{Br}^{\prime}$ for a torus (cf. [ELEN-NARA]), the Corollary to Theorem 1 applies, and we have proved Theorem 2 for $n \geqslant 2 g-1$.

Second step. We assume $3 \leqslant n \leqslant 2 g-2$. Choose $x_{0} \in C$ and interpret symmetric products of $C$ as spaces of effective divisors on $C$. Then for any integer $N \geqslant 1$, we have an embedding

$$
j: C^{(N-1)} \rightarrow C^{(N)}, \quad D \mapsto D+x_{0} .
$$

Recall [MAC, (12.2)] that the corresponding map in cohomology $H^{2}(j)$ : $H^{2}\left(C^{(N)}, \mathbf{Z}\right) \rightarrow H^{2}\left(C^{(N-1)}, \mathbf{Z}\right)$ is an isomorphism for $N \geqslant 4$.

Starting from the short exact sequence

$$
0 \rightarrow \mathbf{Z} \stackrel{r}{\rightarrow} \mathbf{Z} \stackrel{\exp (2 i \pi / r .)}{\rightarrow} \mu_{r} \rightarrow 1
$$

we get a commutative diagram

$$
\begin{array}{ccc}
H^{2}\left(C^{(N)}, \mathbf{Z}\right) & \rightarrow & H^{2}\left(C^{(N)}, \mu_{r}\right) \\
\imath \downarrow H^{2}(j) & & \downarrow H^{2}\left(j, \mu_{r}\right) \\
H^{2}\left(C^{(N-1)}, \mathbf{Z}\right) & \rightarrow & H^{2}\left(C^{(N-1)}, \mu_{r}\right) \rightarrow H^{3}\left(C^{(N-1)}, \mathbf{Z}\right) .
\end{array}
$$

Another result of Macdonald [MAC, (12.3)], is that the cohomology groups $H^{\mu}\left(C^{(\nu)}, \mathbf{Z}\right)$ are all torsion-free. On the other hand, $H^{2}\left(C^{(N-1)}, \mu_{r}\right)$ is a torsion group, and $H^{2}(j)$ is an isomorphism for $N \geqslant 4$. So the above diagram shows that the maps

$$
H^{2}\left(j, \mu_{r}\right): H^{2}\left(C^{(N)}, \mu_{r}\right) \rightarrow H^{2}\left(C^{(N-1)}, \mu_{r}\right)
$$

are surjective for $N \geqslant 4$.

Hence, the composed map $H^{2}\left(C^{(2 g-1)}, \mu_{r}\right) \rightarrow H^{2}\left(C^{(n)}, \mu_{r}\right)$ is also surjective for $n \geqslant 3$. Recalling Remarks 2 and 1 , we see that Theorem 2 is true for $3 \leqslant n \leqslant 2 g-2$, since it is true for $n=2 g-1$ (by the first step).

Third step. The theorem being obvious for $n=1$, the only remaining case is $n=2$. Let $j$ denote the embedding $C^{(2)} \rightarrow C^{(3)}$. According to [MAC, (12.2)], we have an exact sequence of abelian groups

$$
0 \rightarrow H^{2}\left(C^{(3)}, \mathbf{Z}\right) \stackrel{H^{2}(j)}{\rightarrow} H^{2}\left(C^{(2)}, \mathbf{Z}\right) \rightarrow A \rightarrow 0,
$$

where $A$ is a free abelian group. 
The sequence is, of course, split ( $A$, being free, is projective!), and since $C^{(2)}$ and $C^{(3)}$ have equal second Betti numbers [MAC, (4.2)], $A=0$ and $H^{2}(j)$ is bijective.

We conclude as in the second step, and we have proved Theorem 2 for all $n$.

\section{REFERENCES}

[ELEN] G. Elencwajg, The Brauer group in complex geometry, Brauer Groups in Ring Theory and Algebraic Geometry, Lecture Notes in Math., vol. 917, Springer-Verlag, Berlin, 1982, pp. 222-230.

[ELEN-NARA] G. Elencwajg and M. S. Narasimhan, Projective bundles on a complex torus, J. Reine Angew. Math. 340 (1983), 1-5.

[IGAB] O. Gabber, Some theorems on Azumaya algebras, Lecture Notes in Math., vol. 844, SpringerVerlag, Berlin and New York, 1981, pp. 129-209.

[GROT] A. Grothendieck, Le groupe de Brauer. I, II, III, Dix Exposés sur la Cohomologie des Schémas, Masson, Paris, and North-Holland, Amsterdam, 1968.

[HOOB] R. Hoobler, Brauer groups of abelian schemes, Ann. Sci. Ecole. Norm. Sup. (4) 5 (1972), 45-70.

[MAC] I. G. Macdonald, Symmetric products of an algebraic curve, Topology 1 (1962), 319-343.

I.M.S.P., Laboratoire AssociE 168, Parc Valrose, 06034 Nice - Cedex, France 